Original paper

\title{
The occurrence of autoantibodies in patients with chronic HCV infection, including patients dialyzed and after kidney transplantation
}

\author{
Tadeusz W. Łapiński, Magdalena Rogalska-Płońska, Anna Parfieniuk-Kowerda, Magdalena Świderska, Robert Flisiak
}

Department of Infectious Diseases and Hepatology, Medical University of Białystok, Poland

\begin{abstract}
Introduction: There are reports suggesting that hepatitis $\mathrm{C}$ virus (HCV) may stimulate the autoimmune process. Studies have been undertaken to evaluate the occurrence and type of autoantibodies in HCV-infected patients with and without immunosuppression. Results were analyzed according to HCV genotype, intensity of inflammation and liver fibrosis stage.

Material and methods: The study included 105 patients chronically infected with HCV, including 25 with immunological suppression administered for kidney disease or kidney transplantation. Blood samples were tested by immunoblotting for the presence of AMA-M2, SLA/LP, LKM-1, LC1, anti-F-actin, anti-desmin, anti-myosin, anti-gp210 and anti-sp100 autoantibodies, and ANA. All the patients were scored for autoimmune hepatitis.

Results: Autoantibodies were detected in $32.5 \%$ of patients without immunosuppression and in $16 \%$ with immunosuppression. Single types of autoantibodies were identified in 26\% of patients. The most frequent ones were ANA (19\%) and AMA-M2 (5.7\%). The presence of antibodies in patients with genotype 1 was significantly higher in comparison to their occurrence in genotype 3. Autoimmune hepatitis was not diagnosed in any of the patients. Immunoglobulin $\mathrm{G}$ level was significantly higher in patients with detectable autoantibodies, compared to patients without antibodies (1.89 vs. $1.28 \mathrm{~g} / \mathrm{dl}, p<0.001$ ). No correlation between fibrosis stage or intensity of inflammatory state and the frequency of antibodies was found.

Conclusions: The antibodies are significantly more frequent in patients without immunosuppression and in patients infected with genotype 1 than genotype 3 . The presence of these autoantibodies is not associated with the development of autoimmune hepatitis. Higher level of immunoglobulin $\mathrm{G}$ in the serum correlates with the presence of autoantibodies.
\end{abstract}

Key words: autoantibodies, HCV infection, immunosuppressive therapy.

\section{Address for correspondence}

Prof. Tadeusz W. Łapiński, Department of Infectious Diseases and Hepatology, Medical University of Bialystok, 14 Żurawia St., 15-540 Bialystok, Poland, e-mail: twlapinski@gmail.com

\section{Introduction}

Hepatitis C virus (HCV) infection is a common cause of chronic inflammatory liver injury, but not only that. Hepatitis $\mathrm{C}$ virus infection may influence the composition of intracellular proteins. This fact is connected with the binding of viral proteins to host proteins, resulting in the change of their spatial and biochemical structure. Moreover, viral infections may influence production of proteins structurally similar to those naturally occurring, but showing antigenic differences. This phenomenon, called molecular mimicry, constitutes one of the most important theories explaining the existence of autoimmune diseases. Presentation of proteins modified according to this mechanism may be responsible for the development of autoimmune processes leading to occurrence of different types of autoantibodies [1]. Autoantibodies are not the only active element in autoimmune processes. Actually, autoantibodies are readily available markers 
Table 1. Control and study groups

\begin{tabular}{lccc}
\hline Factor & HCV without immunosuppression & HCV with immunosuppression & Control group \\
\hline Number of patients & 80 & 25 & 27 \\
\hline Age (years) & $44(23-67)$ & $46(23-69)$ & $37(25-56)$ \\
\hline Female/male (\%) & $30 / 50(38 / 62)$ & $11 / 14(44 / 56)$ & $13 / 14(48 / 52)$ \\
\hline Genotype $1 / 3(\%)$ & $58 / 22(73 / 27)$ & $21 / 4(84 / 16)$ & - \\
\hline
\end{tabular}

There were no significant differences between the groups.

of activation of such processes, but cannot be used as a definitive predictor of autoimmune disease, such as autoimmune hepatitis (AIH). Immunization by HCV may lead to autoimmune hepatitis, which overlaps with viral hepatitis [2]. This is extremely important from the therapeutic point of view, especially among patients with concurrent injuries to other organs, such as chronic kidney injury or kidney transplantation.

This study aimed to evaluate the frequency and type of autoantibodies among patients taking immunosuppressive therapy for chronic kidney injury or patients after kidney transplantation, compared to patients infected with HCV, in whom immunosuppression was not used. Occurrence of autoantibodies was correlated with HCV genotype, intensity of inflammatory state and the stage of fibrosis in the hepatic tissue. The relation between the presence of autoantibodies and the level of immunoglobulin G, $\alpha$-fetoprotein (AFP) and guanosine- $5^{\prime}$-triphosphate (GTP) in the serum was tested. All the patients were scored for AIH.

\section{Material and methods}

The study included one hundred and five patients infected with $\mathrm{HCV}$, i.e. 79 infected with genotype $1 \mathrm{~b}$ and 26 with genotype $3 \mathrm{a}$. None of the patients was previously treated with antiviral therapy. Immunosuppressive therapy was used in 25 patients: 19 patients with end-stage renal disease (glomerulonephritis) and 6 after kidney transplantation, also due to being previously diagnosed with glomerulonephritis (Table 1). In 5 patients after kidney transplantation cyclosporine was administered, and in 1 tacrolimus. Fourteen patients with end-stage renal failure were hemodialyzed and periodically given glucocorticosteroids. Five patients with end-stage renal failure, who were not dialyzed, were constantly taking glucocorticosteroids.

Study inclusion criteria included diagnosis of chronic hepatitis C, exclusion of HBV and HIV, and no autoimmune hepatitis diagnosed in patient history.

The control group included 27 healthy volunteers.

Hepatitis $C$ virus infection was diagnosed based on the presence of HCV RNA in the serum. The study was was performed using RT-PCR with starters specific for a non-coding viral $5^{\prime}$-terminal genomic fragment (5'UTR). Viral genotype was determined with direct sequencing of the product obtained in the PCR reaction (Syngen Biotech, USA) ${ }^{1}$.

The presence of autoantibodies in the serum was detected with Liver-9-line test, by immunoblotting (membrane-fixed immunoblot test) purchased from Organtec, Germany. In the test, processed serum was transferred onto a nitrocellulose plate containing antigens binding antibodies against F-actin, desmin, myosin, liver cytosol antigen type 1 (LC1), liver-kidney microsome type 1 (LKM-1), soluble liver antigen/ liver-pancreas (SLA/LP) antigens, Sp100, gp210 and antimitochondrial type M2 antibodies (AMA-M2). Moreover, antinuclear antibodies (ANA) were tested in the serum by ELISA, using a BEP $2000 \mathrm{~A} 2 / \mathrm{V}$ instrument by Siemens ${ }^{2}$.

All patients were tested for HBV infection by evaluating the presence of HBs antigen in the serum, and for HIV by measuring anti-HIV antibodies (Abbott, USA).

All patients were evaluated for the level of gamma immunoglobulin, AFP and GTP activity in the serum².

Fibrosis was measured in 16/25 (64\%) immunosuppressed patients: in 6 liver biopsy was performed, and in 10 elastography (Aixplorer, Supersonic). From among 80 patients without immunosuppression, liver biopsy was performed in 58, and elastography in 22 patients. In patients subjected to liver biopsy, bioptates were stained with hematoxylin and eosin in order to determine the intensity of the inflammatory state, and with Syrian red to visualize connective tissue. Morphological state of the liver was described according to the Scheuer classification ${ }^{3}$. Results of liver fibrosis

${ }^{1}$ Analysis was performed at the Clinical Molecular Biology Department, Medical University of Bialystok, Head: Prof. Nikliński.

${ }^{2}$ Tests were performed in the Laboratory of K. Dluski Memorial Regional Specialist Hospital in Bialystok, Head: Jolanta Czajkowska, MSc.

${ }^{3}$ Morphological state of liver bioptates was evaluated by Prof. A. Panasiuk at the Clinics of Infectious Diseases and Hepatology, Medical University of Bialystok. 
determined by elastography were denoted in $\mathrm{kPa}$ and automatically converted into the Metavir scale.

After approval of the study by the Bioethical Committee at the Medical University of Bialystok, the patients expressed informed consent to participation in the research.

Statistical analysis was performed with $\chi^{2}$, Student's $t$ and Mann-Whitney $U$ tests. Significance was established at $p<0.05$.

\section{Results}

The presence of autoantibodies was found in 4/25 (16\%) immunosuppressed patients infected with HCV and in 32/80 (40\%) patients without immunosuppression (the difference was statistically significant, $p=0.001)$. In none of the patients with kidney injury or after kidney transplantation, and in all of those with diagnosed chronic glomerulonephritis, neither ANA, AMA-M2 nor LKM antibodies were found. Only single types of autoantibodies were detected in the group of patients receiving immunosuppression. Among the patients without immunosuppression, single types of autoantibodies were found in 21 patients, in 4 patients simultaneous presence of two autoantibodies was detected, and in one patient 3 autoantibodies. Among 27 healthy volunteers from the control group, autoantibodies were not detected (Fig. 1).

Antibodies against F-actin, LC-1 and sp- 100 protein were not detected in any patient. The most frequently found antibodies were ANA $(20 / 105 ; 19 \%)$ and AMA-M2 (6/105; 5.7\%).

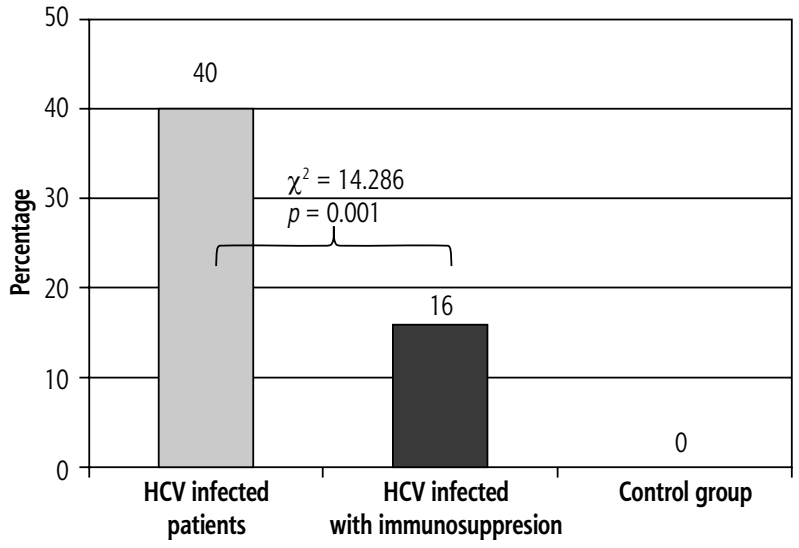

Fig. 1. Prevalence of autoantibodies in particular groups of patients

In the group of patients without immunosuppression there was a significant difference in the frequency of autoantibodies in patients infected by genotype 1 compared to those infected with genotype 3 ( $46.5 \%$ vs. $22.7 \%, p=0.001$ ) (Table 2).

Scoring for autoimmune hepatitis was performed in the group of patients with detected autoantibodies. Evaluation was performed according to the guidelines of the International Autoimmune Hepatitis Group, where the minimal number of points indicating autoimmune disease is 15 [3]. The highest calculated number of points among the studied patients was 7 (HLA DR3 or DR4 was not taken into consideration when scoring, but omission of this factor may decrease the total result to 14 points).

Level of immunoglobulin $G$ in the serum was significantly higher in patients with autoantibodies, com-

Table 2. Type of autoantibodies depending on the genotypes of the virus among HCV infected patients

\begin{tabular}{|c|c|c|c|c|c|c|}
\hline \multirow[t]{3}{*}{ Autoantibodies } & \multicolumn{3}{|c|}{ Patients without immunosuppression } & \multicolumn{3}{|c|}{ Patients with immunosuppression } \\
\hline & Genotype $1(n=58)$ & Genotype $3(n=22)$ & Total $(n=80)$ & Genotype $1(n=21)$ & Genotype $3(n=4)$ & Total $(n=25)$ \\
\hline & $n(\%)$ & $n(\%)$ & $n(\%)$ & $n(\%)$ & $n(\%)$ & $n(\%)$ \\
\hline ANA & $15(25.9)^{x}$ & $2(9.1)^{x}$ & $17(21.3)$ & $3(14)$ & 0 & $3(12)$ \\
\hline Anti-Myosin & $2(3.4)$ & $1(4.5)$ & $3(3.7)$ & 0 & 0 & 0 \\
\hline Anti-Desmin & $2(3.4)$ & 0 & $2(2.5)$ & 0 & 0 & 0 \\
\hline Anti-F-actin & 0 & 0 & 0 & 0 & $1(25)$ & $1(4)$ \\
\hline LKM & $1(1.7)$ & 0 & $1(1.3)$ & 0 & 0 & 0 \\
\hline SLA/LP & $1(1.7)$ & 0 & $1(1.3)$ & 0 & 0 & 0 \\
\hline gp120 & $2(3.4)$ & 0 & $2(2.5)$ & 0 & 0 & 0 \\
\hline AMA-M2 & $4(6.9)$ & $2(9.1)$ & $6(7.5)$ & 0 & 0 & 0 \\
\hline Total & $27(46.5)^{*}$ & $5(22.7)^{*}$ & $32(40.0)^{\#}$ & $3(14)$ & $1(25)$ & $4(16)^{\#}$ \\
\hline
\end{tabular}

xStatistically significant difference $(p<0.05)$

"Statistically significant difference $(p<0.05)$

"Statistically significant difference $(p<0.05)$ 


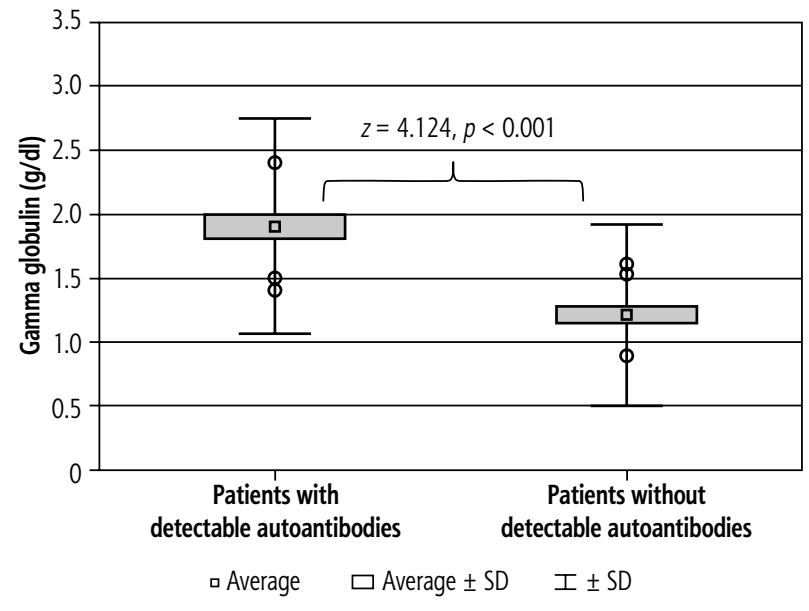

Fig. 2. Serum $\lg G$ concentration in the study groups

pared to patients without autoantibodies $(1.89 \pm 1.82 \mathrm{vs}$. $1.28 \pm 1.2 \mathrm{~g} / \mathrm{dl} ; Z=4.124 ; p<0.001$ ) (Fig. 2 ). It has been demonstrated that $\operatorname{IgG}$ concentration above $1.6 \mathrm{~g} / \mathrm{dl}$ is associated with the presence of autoantibodies, and is found in $87 \%$ of such patients.

No difference in AFP level or GPT activity was found among the patients with or without the presence of autoantibodies.

Average values of fibrosis in immunosuppressed patients were comparable to fibrosis patients without immunosuppression (average $1.78 \pm 1.12$ vs. $1.88 \pm 1.03$; $p=0.78)$.

There was no difference in the progression of fibrosis in patients with the presence of autoantibodies compared to patients without autoantibodies $(2.32 \pm 0.94$ vs. $2.60 \pm 0.56, p=0.36$ ).

\section{Discussion}

The influence of the liver on the mechanisms of tolerance and hyperreactivity is very important for the development of autoimmune diseases, autoimmune hepatitis in particular. Homology of HCV antigens' structure and the structure of hepatocyte proteins allows for consideration of the possibility of autoaggression as the effect of cross-antigenicity, leading to the development of autoimmune hepatitis.

Antinuclear antibodies (ANA) are directed against deoxyribonucleic acid and deoxyribonucleoproteins contained in the cell nucleus. Antinuclear antibodies are usually associated with autoimmune hepatitis type 1 . In their study performed on 4754 adult Americans aged over 12, Satoh et al. detected ANA in 13\% [4]. However, the occurrence of ANA in the healthy population is probably dependent on many factors, including geographical distribution. Retrospective analysis performed by Khairy et al. among 3673 Egyptians infected with HCV and among healthy people demonstrated the presence of ANA in both of these groups in similar proportions, around $1.6 \%$ [5]. In a study by Molazadeh et al., ANA were present in 0.9\% (5/560) of healthy people in Iran [6]. In our research, in the control group of 27 persons we did not detect these autoantibodies, but among those infected with HCV they were detected in $19 \%$ of patients. Data presented by Basile et al. point to significantly more frequent occurrence of ANA in $65 \%$ of people infected with $\mathrm{HCV}$, compared to healthy people [7]. It seems that HCV activates mainly the process of ANA synthesis.

According to the international diagnostic criteria of autoimmune hepatitis (International Autoimmune Hepatitis Group) [3, 8], AIH diagnosis could not be established in any of our patients. Among the patients with immunosuppression, autoantibodies were much less frequently found than in patients without immunosuppression. These observations point to activation of autoimmune mechanisms by HCV, but not to the influence on advanced immunological changes leading to the development of autoimmune diseases.

AMA-M2 autoantibodies, the second most frequently occurring type of autoantibodies among patients infected with $\mathrm{HCV}(7 \%)$, are characteristic in patients with primary biliary cirrhosis (PBC). Two subtypes of AMA antibodies, one against the proteins of cellular membrane pores gp210, and another against nuclear specific protein sp100, are currently thought to be more specific in the diagnostics of $\mathrm{PBC}$ than AMA-M2. Compared to AMA-M2, anti-gp210 and anti-SP100 are better markers of PBC exacerbation and risk of PBC development [9]. Thus, detection of these antibodies in only 2 patients seems justified. AMA-M2 are directed against mitochondrial internal membrane proteins. They are rarely detected in patients infected with $\mathrm{HCV}$ and usually occur in women during the treatment with interferon [10].

Antibodies directed against F-actin belong to the group of antibodies against smooth muscles. They may be produced in response to changes of filaments in the cells of the biliary tract and hepatic tissue matrix. Studies suggest an association of these proteins with HCV replication and formation of neoplastic lesions [11]. Hudacko et al. demonstrated F-actin antibodies in $16 \%$ of patients infected with HCV or HCV/HIV. Moreover, the authors documented the correlation between occurrence of these antibodies and severity of the inflammatory state in the liver [12]. In our research, antibodies directed against F-actin were not found in any of the patients.

Only in 3 patients were anti-myosin antibodies, usually occurring in patients with cardiac diseases, found. Antibodies against desmin were detected rarely, i.e. only 
in 2 patients. Although desmin is a protein building Ito cells, such antibodies are found sporadically in autoimmune liver diseases [13].

Anti-SLA antibodies are thought to be a marker of AIH type III [14]. In our research these antibodies were found in one person, which agrees with reports suggesting that they are rarely found among people infected with HCV [15].

Antibodies against microsomal proteins of liver and kidneys, anti-LKM-1, are a marker of AIH type II [16]. It is suggested that these antibodies are formed mainly through molecular mimicry connected with the loss of immunological tolerance. The change of the structure of autoantigens, as the result of binding viral proteins with host proteins and forming a complex significantly differing from the primary antigen, usually underlies this phenomenon [17]. In our research the presence of these antibodies was demonstrated in only one person, which confirms the stimulating effect of the virus on autoimmune processes, but not the ability to initiate an autoimmune disease.

It is thought that the course of chronic HCV infection among dialyzed patients is more aggressive compared to the patients with healthy kidneys. However, clinical observations show a similar clinical picture and individual progress of the disease, regardless of dialysis therapy and other significant kidney injuries $[18,19]$. Separate consideration of patients taking immunosuppressive agents because of kidney injury or after kidney transplantation in our studies aimed at confirming an inhibitory effect of immunosuppression on autoimmunization processes. We did manage to demonstrate such an effect in our research. However, such an effect is not important from the point of view of the patient, because the autoimmunization process, not leading to the development of autoimmune hepatitis, does not significantly influence the clinical course of HCV infection. Our results confirm the importance of immunological suppression in the absence of stimulation of the immune system. This is important because in any patients (even with positive autoantibodies) is not obtained AJH score and identifiable autoimmune disease.

The studies by Marconcini et al. suggest that the presence of autoantibodies (especially non-organ specific antibodies - NOSA) correlates with worse clinical course of chronic hepatitis type $\mathrm{C}$ and exacerbation of inflammatory changes in the liver [20]. Our research does not confirm such observations. Also the study by Mauss et al., which analyzed the influence of the presence of autoantibodies on the efficacy of antiviral treatment among over 24 thousand patients infected with $\mathrm{HCV}$, did not find any negative effect of ANA, LKM,
SMA or AMA on the clinical state and effect of antiviral treatment. In the opinion of the authors, routine testing for autoantibodies, before the treatment of patients with HCV, is redundant [21]. The results of our research seem to be in line with this statement, since they demonstrate that autoimmune diseases do not develop among patients infected with $\mathrm{HCV}$.

\section{Conclusions}

The antibodies are significantly more prevalent in patients without immunosuppression and in patients infected with genotype 1 than genotype 3 . Use of immunosuppression significantly reduces the incidence of autoantibodies. Autoimmune stimulation by HCV appears to be insufficient for development of autoimmune hepatitis.

\section{Disclosure}

Authors report no conflict of interest.

\section{References}

1. Narciso-Schiavon JL, Schiavon L de L. Autoantibodies in chronic hepatitis C: A clinical perspective. World J Hepatol 2015; 7: 1074-1085.

2. Acay A, Demir K, Asik G, et al. Assessment of the Frequency of Autoantibodies in Chronic Viral Hepatitis. Pak J Med Sci 2015; 1: $150-154$

3. Manns MP, Czaja AJ, Gorham JD, et al. American Association for the Study of Liver Diseases. Diagnosis and management of autoimmune hepatitis. Hepatology 2010; 51: 2193-2213.

4. Satoh M, Chan EK, Ho LA, et al. Prevalence and sociodemographic correlates of antinuclear antibodies in the United States. Arthritis Rheum 2012; 64: 2319-2327.

5. Khairy M, El-Raziky M, El-Akel W, et al. Serum autoantibodies positivity prevalence in patients with chronic HCV and impact on pegylated interferon and ribavirin treatment response. Liver Int 2013; 33: 1504-1509.

6. Molazadeh M, Karimzadeh H, Azizi MR. Prevalence and clinical significance of antinuclear antibodies in Iranian women with unexplained recurrent miscarriage. Iran J Reprod Med 2014; 3: 221-226.

7. Basile U, Gulli F, Torti E, et al. Anti-nuclear antibody detection in cryoprecipitates: distinctive patterns in hepatitis $C$ virus-infected patients. Dig Liver Dis 2015; 1: 50-56.

8. Hennes EM, Zeniya M, Czaja AJ, et al. International Autoimmune Hepatitis Group. Simplified criteria for the diagnosis of autoimmune hepatitis. Hepatology 2008; 48: 169-176.

9. Hu SL, Zhao FR, Hu Q, et al. Meta-analysis assessment of GP210 and SP100 for the diagnosis of primary biliary cirrhosis. PLoS One 2014; 7: e101916.

10. Macaluso FS, Alessi N, Cabibi D. Antimitochondrial antibody $-\mathrm{M} 2$ positive autoimmune hepatitis during standard of care for chronic hepatitis C. Hepatol Res 2012; 4: 428-432.

11. Oikonomou KG, Zachou K, Dalekos GN. Alpha-actinin: a multidisciplinary protein with important role in B-cell driven autoimmunity. Autoimmun Rev 2011; 7: 389-396. 
12. Hudacko RM, Alvarez GA, Talal AH, et al. Clinical and biologic importance of F-actin autoantibodies in HCV monoinfected and HCV-HIV coinfected patients. Am J Clin Pathol 2010; 2: 228-234.

13. Mabuchi A, Mullaney I, Sheard P. Role of Hepatic Stellate Cells in the Early Phase of Liver Regeneration in Rat: Formation of Tight Adhesion to Parenchymal Cells. Comp Hepatol 2004; 6: 910-916.

14. Czaja AJ. Autoantibodies as Prognostic Markers in Autoimmune Liver Disease. Dig Dis Sci 2010; 8: 2144-2161.

15. Penalva Polo JC, Verdú Benavente C, Belda Abad G, et al. Persistent acute hepatitis in a patient with chronic hepatitis C. Gastroenterol Hepatol 2006; 8: 451-454.

16. Invernizzi P, Mackay IR. Autoimmune liver diseases. World J Gastroenterol 2008; 21: 3290-3291.

17. Mocumbi AO, Latif N, Yacoub MH. Presence of circulating anti-myosin antibodies in endomyocardial fibrosis. PLoS Negl Trop Dis 2010; 4: e661.

18. Ozer Etik D, Ocal S, Boyacioglu AS. Hepatitis C infection in hemodialysis patients: A review. World J Hepatol 2015; 6: 885-895.

19. Carvalho-Filho RJ, Feldner AC, Silva AE, et al. Management of hepatitis $\mathrm{C}$ in patients with chronic kidney disease. World J Gastroenterol 2015; 2: 408-422.

20. Marconcini ML, Fayad L, Shiozawa MB, et al. Autoantibody profile in individuals with chronic hepatitis C. Rev Soc Bras Med Trop 2013; 46: 147-153.

21. Mauss S, Berger F, Schober A, et al. Screening for autoantibodies in chronic hepatitis $C$ patients has no effect on treatment initiation or outcome. J Viral Hepat 2013; 4: e72-77. 